\title{
Summary of the Primary Care Management of Hepatitis B - Quick Reference
}

\author{
Gale-Rowe $M^{1 *}$, Latham-Carmanico $C^{1}$, Lalonde $F^{1}$ and Wong $T^{1}$ on behalf of the authors and contributors \\ 1 Public Health Agency of Canada, Ottawa, ON \\ * Corresponding author: Margaret.galerowe@phac-aspc.gc.ca
}

\section{Introduction}

Hepatitis $B(\mathrm{HB})$ virus is a highly infectious vaccine-preventable disease. Canada is a region of low endemicity; however, certain vulnerable populations, including Aboriginal peoples, men who have sex with men, streetinvolved youth and people who are or have been incarcerated, are disproportionately affected. The low prevalence is mainly attributable to universal HB immunization programs, which are available and accessible in all provinces and territories.

In Canada, an estimated 300,000 individuals are chronically infected with HB, which represents less than $1 \%$ of Canadians. Data suggest that of those affected with chronic HB, $70 \%$ are immigrants from highly endemic regions. Two thirds of chronically infected people are unaware of their status. In the absence of appropriate intervention $15 \%$ to $40 \%$ of them will have long-term sequelae of HB, such as cirrhosis, end-stage liver disease and/or hepatocellular carcinoma, and in addition can infect others There is an urgent need to screen, diagnose and treat, where appropriate, chronic HB infection in order to reduce the associated morbidity and mortality and to prevent further transmission.

The objective of the Primary Care Management of Hepatitis B-Quick Reference (1) is to assist primary care and public health practitioners in the prevention, screening and management of $\mathrm{HB}$ in patients at risk of or infected with HB. This document provides a brief summary.

The Quick Reference does not supersede any provincial/territorial legislative, regulatory, policy and practice requirements or professional guidelines that govern and inform the practice of care providers in their respective jurisdictions.

\section{Methods}

The Quick Reference was developed by the Public Health Agency of Canada in collaboration with working group members with expertise in HB, HIV and related co-infections. The content of this clinical resource reflects current published information and evidence-based resources, such as the Management of Chronic Hepatitis B: Canadian Association for the Study of the Liver Consensus Guidelines (2) and was tailored on input from expert hepatologists, laboratory specialists, public health practitioners, physicians and nurses.

\section{Results}

The content of the Quick Reference is divided into 11 modules and includes links to credible resources to support the most recent immunization, treatment and other management recommendations. Table 1 identifies the titles of all the modules and provides a short summary of the content available in each module. 
Table 1. Highlights of the Primary Care Management of Hepatitis B - Quick Reference

\begin{tabular}{|c|c|}
\hline Modules & Highlights \\
\hline 1. Who Should Be Tested for HB? & $\begin{array}{l}\text { Screen routinely at first visit anyone who has risk factors } \\
\text { for HB (e.g. blood exposure; high risk sexual behaviours; } \\
\text { intravenous drug use; incarceration; and travel to/former } \\
\text { residence in an endemic country), and screen routinely } \\
\text { anyone who is pregnant, has Hepatitis C, HIV or other } \\
\text { immunocompromising condition or is planning to take an } \\
\text { immunosuppressive agent. In addition, clinicians should } \\
\text { test anyone with signs and symptoms of acute hepatitis } \\
\text { or clinical and laboratory findings suggestive of liver } \\
\text { disease. }\end{array}$ \\
\hline 2. Approach to HB Screening and Testing & $\begin{array}{l}\text { The most commonly used HB serologic markers are the } \\
\text { surface antigen (HBsAg), the surface antibody (anti-HBs) } \\
\text { and the core antibody (anti-HBc), either to detect } \\
\text { infection or to determine immune status. Other HB } \\
\text { serologic markers exist for screening in patients with } \\
\text { defined clinical conditions or for testing to confirm } \\
\text { diagnosis in patients with acute hepatitis. Note: the } \\
\text { choice of tests should be based on patient history and } \\
\text { clinical presentation. }\end{array}$ \\
\hline
\end{tabular}

3. Interpretation of HB Diagnostic Test Results

Given the various HB diagnostic tests and serologic markers available, interpretation of results can be complex and, where appropriate, the guidance of a specialist should be sought. A table provides guidance for the interpretation of the different $\mathrm{HB}$ serologic test results and recommended actions.

\section{Initial Management of Patients with HBsAg- Positive Results}

An algorithm highlights the responsibilities of both primary care and public health. The primary care provider advises the patient of the diagnosis and reports to local public health. An algorithm lists specific actions on follow-up for both the primary care provider and the public health department, and also highlights the importance of ongoing communication between the two parties to achieve proper case management and followup.

\section{Natural History and Management of Acute HB}

\section{Initial Evaluation of Confirmed Chronic HB}

Acute $\mathrm{HB}$ does not require antiviral treatment, as $95 \%$ of immunocompetent adults will recover within 6 months and develop lifelong immunity. The management of acute HB includes relief of symptoms, if any, monitoring and prevention of hepatic complications, as well as counselling aimed at preventing transmission. The module outlines baseline laboratory testing and lists indications for repeat testing to confirm/rule out chronic infection. It also lists indications for urgent referral to a specialist of patients with a severe presentation.

Provides guidance on baseline clinical evaluation and initial laboratory/imaging evaluation of patients with confirmed chronic infection, and lists indications for urgent and semi-urgent referral to a specialist. 


\section{Natural History of Chronic HB}

The natural history and progression of chronic $\mathrm{HB}$ is complex and non-linear, and varies from person to person. Familiarity with the different phases can help guide decisions related to treatment and monitoring. The module describes the phases of chronic HB and the associated serologic markers, and explains their relations to liver inflammation, histologic activity, degree of fibrosis and the risk of progression to cirrhosis and hepatocellular carcinoma. The module includes a schematic visualization of the different phases of chronic HB.

\section{Long-Term Management of Confirmed Chronic HB}

Outlines the recommended frequency of serologic and histologic monitoring, the goal being to prevent progression to cirrhosis, hepatocellular carcinoma and liver decompensation. It is recommended that certain patients who meet the outlined criteria undergo lifelong screening for hepatocellular carcinoma at 6-month intervals by means of abdominal ultrasound. A table of suggested follow-up by phase of infection as determined by serologic and histologic findings is also available.

\section{Treatment of Chronic HB and Monitoring of Patients on Treatment}

The module does not provide treatment options/regimens. Practitioners are referred to the Management of Chronic Hepatitis B: Canadian Association for the Study of the Liver Consensus Guidelines 2012.

Not all patients with chronic $\mathrm{HB}$ infection require treatment; the decision to treat depends on several factors.

The current approved treatments for $\mathrm{HB}$ are interferon injections or oral nucleoside/nucleotide analogues, all of which should be initiated by a hepatologist or other physician with experience in the management of viral hepatitis (e.g. an infectious disease specialist). The module provides advice on the decision to treat, treatment initiation and the duration of therapy. It also provides guidance for ongoing monitoring of patients receiving treatment and indications for follow-up with a specialist.

\section{Prevention and Vaccination Checklist}

Provides a link to details on publicly funded $\mathrm{HB}$ vaccine programs in Canada. It includes a checklist that prompts practitioners to discuss and offer vaccine to patients, as per the recommendations in the Canadian Immunization Guide. Special attention is paid to pregnant women. If a pregnant woman tests positive for $\mathrm{HB}$, practitioners are advised to refer her to a specialist before the third trimester of pregnancy for guidance on indications for/timing of treatment initiation to prevent vertical transmission. The module also provides guidance on when treatment can be discontinued postpartum; however, it should be noted that the discontinuation of treatment is only applicable if the mother does not require ongoing therapy for $\mathrm{HB}$.

Provides practitioners with guidance on counselling for all patients to reduce the risk of transmission, including advice on disclosure, and specific advice for pregnant women and patients with acute HB. The module also provides general guidance to help patients with chronic $\mathrm{HB}$ reduce their risk of liver damage and specific advice for all patients, including those with cirrhosis, on maintaining a healthy lifestyle. 


\section{Discussion}

The Quick Reference is a very useful and practical clinical resource for primary care and public health professionals, as it guides them through all the main steps for the complete management of HB and refers them to credible resources already available for treatment, immunization and management recommendations.

\section{References}

(1) Primary Care Management of Hepatitis B - Quick Reference: Public Health Agency of Canada;

2013. http://www.phac-aspc.gc.ca/publicat/hep/hbv-vhb/index-eng.php

(2) Coffin CS, Fung SK, Ma MM. Management of chronic hepatitis B: Canadian Association for the Study of the Liver consensus guidelines. Can J Gastroenterol. 2012 Dec; 26(12): 917-38.

\section{Acknowledgements}

Many thanks to the authors:

Andonov A, Ling R, Baril JG, Myers R, Brubacher C, Osiowy C, Butler G, Pritchard LM, Gale-Rowe M, Verkoeyen J, Heathcote J, Yim C, Latham-Carmanico C.

Many thanks to the contributors, the Centre for Communicable Diseases and Infection Control and the Centre for Immunization and Respiratory Infectious Diseases at the Public Health Agency of Canada.

\section{Conflicts of interest}

There are no conflicts of interest to declare.

\section{Funding}

This project was funded by the Public Health Agency of Canada. 